\title{
COVID-19 and Re-Commencement of Early Phase Clinical Trials in Respiratory Medicine
}

\author{
Aernout D van Haarst ${ }^{1}{ }^{*}$, David Bell ${ }^{1}$, Sunu Valasseri ${ }^{1}$, J Stuart Elborn ${ }^{2}$ and Damian G Downey ${ }^{3}$ \\ ${ }^{1}$ Celerion, Belfast, UK \\ ${ }^{2}$ Faculty of Medicine, Health and Life Sciences, Queen's University Belfast, UK \\ ${ }^{3}$ Wellcome-Wolfson Institute of Experimental Medicine, Queen's University Belfast, UK.
}

Submission: July 16, 2021; Published: July 28, 2021

*Corresponding author: Aernout D van Haarst, Celerion, 22-24 Lisburn Road, BT9 6AD, Belfast, UK

\section{Abstract}

COVID-19 particularly impeded the conduct of early phase, respiratory drug trials, which relates to the intensity and complexity of the required study procedures. We describe how the implementation of risk mitigation measures helped overcome challenges to their conduct.

Keywords: COVID-19; Respiratory; Early phase; Clinical trial; Clinical pharmacology

Abbreviations: CF: Cystic Fibrosis; COVID-19: Coronavirus Disease 2019; ERS: European Respiratory Society; PCR: Polymerase Chain Reaction; PPE: Personal Protective Equipment; SARS-CoV-2: Severe Acute Respiratory Syndrome Coronavirus-2

\section{Short Communication}

Upon the start of the Coronavirus Disease 2019 (COVID-19) pandemic, an urgent need for new treatments and vaccines developed, but, paradoxically, the pandemic has also impacted clinical trial conduct. The impact of the pandemic has in particular been tangible for early phase trials in respiratory medicine.

Firstly, because lung function testing procedures carry a risk of spreading SARS-CoV-2 through aerosol generation, the performance of lung function tests has been restricted as an additional risk mitigation measure for respiratory trials. Last year, the European Respiratory Society (ERS) advised that testing procedures for clinical evaluation of patients be limited primarily to spirometry and diffusing capacity, while other tests should only be introduced when risk had been appropriately evaluated, provided that droplet contamination could be contained [1]. For instance, any procedure that is deemed "aerosol generating" requires wearing full Personal Protective Equipment (PPE) including a FFP3 mask.

Secondly, while later stage trials have adopted innovative solutions allowing remote trial activities [2], these approaches offer little added value to early Phase (I/IIa) studies. Typically, early phase respiratory drug trials require intensive pharmacokinetic sampling, frequent and complex pharmacodynamic assessments (e.g., lung function testing), invasive sampling methods (such as bronchoalveolar lavage and induced sputum), respiratory challenge tests and continuous safety monitoring. Therefore, these studies are conducted in specific, early phase clinical trial centres.

Thirdly, people with certain respiratory diseases, may be at a higher risk of Severe Acute Respiratory Syndrome Coronavirus-2 (SARS-CoV-2) infection and/or developing severe respiratory complications [3-6], which may discourage them from trial participation. Early stage trials investigate safety, tolerability and PK of new drug candidates, formulations and routes of administration and explore pharmacological effects and potential drug interactions. Even though early phase trials may include small patient cohorts, they usually offer no personal health benefit to study participants. Yet, exploratory data from patients are crucial for assessment of optimal dose and dosing regimen and for optimizing study designs of confirmatory studies.

With the COVID-19 pandemic, the risk of study participation may have increased due to a risk of infection with SARS-CoV-2. This applies to early phase clinical trials with a need for frequent 
on-site assessments and study procedures with a high risk of aerosol generation and exposure. Consequently, engagement of healthy subjects and patients in early stage trials has become more challenging. In particular, patients with a respiratory disease may not be prepared to take risks by partaking in exploratory trials during the COVID-19 pandemic. However, since the lung physiology of people with lung diseases (with reduced lung function, viscus respiratory secretions and chronic inflammatory states) is different from healthy participants, tolerability, pharmacokinetics, and pharmacodynamics of drugs may also be very different for patients. Hence, there is a critical need to explore drug effects in patients during early drug development.

To enable important early phase clinical research, the potential risk of participation during a pandemic will have to be balanced with the implementation of stringent risk mitigation measures. Previous experience with Phase I studies in patients has been instrumental in dealing with the COVID-19 pandemic in our early phase research center. For example, in cystic fibrosis (CF) studies, special measures for infection control have historically been adopted into our centre, e.g., increasing the stringency of site access and cleaning policies (emphasising high-touch areas) and providing guidance for patient isolation and infection prevention. Such routine and the practical experience from CF clinical trials has helped inform the adaption of trial operations aiming to limit SARS-CoV-2 infection in a respiratory clinical trial population in the following ways.

In response to the COVID-19 pandemic, we have expanded these established infection control measures in line with guidelines issued by major regulatory agencies in response to the pandemic [7-9] and local regulations and drawn up Risk Mitigation Plans for our clinical research facilities. Establishing key risk indicators prior to study start for each protocol is also part of protocol assessment and the risk mitigation process.

In addition, a 3-tiered COVID-19 screening process was introduced for study participants. First, subjects are called to screen for symptoms and contacts with infected people. Second, during on-site screening, symptoms are reviewed, temperature is checked, and a nasopharyngeal swab taken for SARS-CoV-2 polymerase chain reaction (PCR) testing. Finally, the PCR test is repeated at check-in for the actual study visit. Regular, on-site COVID-19 testing is also carried out during study conduct. Staff are checked each working day for temperature, COVID-19 symptoms and contacts with known patients, which is further supported by up to once weekly PCR testing, customized to potential risk of each role. Staff are also advised to get vaccinated against SARS-CoV-2.

Segregated spaces have been created to facilitate social distancing and allow isolation of subjects in case of suspected infection. Moreover, participants and staff wear appropriate, event- and study-specific PPE while at the clinic. Regular hand washing is encouraged and hand sanitizer is available throughout the building.

With preventative measures in place, research facilities have been able to re-start early stage trials. For respiratory drug trials, the challenges have been higher due to the need for aerosol generating procedures. While lung function testing has been resumed in clinical care, the risk/benefit ratio for participants in exploratory trials is substantially different.

In line with ERS guidance, full operation of lung function services can however be resumed if justified in view of local viral prevalence, if reliable screening SARS-CoV-2 is in place and if differential use of PPEs is applied per procedure. With the implementation of strict safety measures, enrolment of healthy volunteers and performance of lung function testing have been re-commenced during the past year. Also, invasive procedures like bronchoalveolar lavage as well as evaluation of patient panels in exploratory trials have been resumed- in our Belfast research center using personally fit PPEs as needed.

With new SARS-CoV-2 variants conceivably looming, COVID-19 may resurge for years and, consequently, pandemicrelated measures will remain in place to allow continuation of clinical trials. The implementation of SARS-CoV-2 screening is reminiscent of the introduction of HIV screening in the 80's into eligibility assessment, yet the measures introduced with COVID-19 go beyond that and dictate more preventative operational settings, especially for respiratory studies.

\section{References}

1. (2020) Lung function testing during COVID-19 pandemic and beyond - Recommendation from ERS Group 9.1 (Respiratory function technologists /Scientists). ERS.

2. Van K-Rietschel S, Dunlevy F, Bulteel V, Downey D, Dupont L (2020) SARS-CoV2 disrupts clinical research: the role of a rare disease-specific trial network. European Respiratory Journal 56(3).

3. Leung JM, Niikura M, Yang CWT, Sin DD (2020) COVID-19 and COPD. European Respiratory Journal 56(2).

4. Shimi G, Zand H (2021) Association of alpha-1-antitrypsin deficiency with vitamin D status: who is most at risk of getting severe COVID-19? Inflamm Res 70(4): 375-77.

5. Kermani NZ, Song WJ, Badi Y, Versi A, Guo Y et al. (2021) Sputum ACE2, TMPRSS2 and FURIN gene expression in severe neutrophilic asthma. Respiratory Research 22(1): 10.

6. Eger K, Bel EH (2021) Asthma and COVID-19: do we finally have answers? Eur Respir J 57(3): 2004451.

7. EC, EMA, HMA (2020) Guidance on the Management of Clinical Trials during the COVID-19 (Coronavirus) Pandemic, Version 4.

8. FDA (2020) Guidance on Conduct of Clinical Trials of Medical Products during COVID-19 Public Health Emergency.

9. MHRA (2020) Guidance: Managing clinical trials during Coronavirus (COVID-19) - How investigators and sponsors should manage clinical trials during COVID-19. 
Your next submission with Juniper Publishers will reach you the below assets

- Quality Editorial service

- Swift Peer Review

- Reprints availability

- E-prints Service

- Manuscript Podcast for convenient understanding

- Global attainment for your research

- Manuscript accessibility in different formats ( Pdf, E-pub, Full Text, Audio)

- Unceasing customer service

Track the below URL for one-step submission https://juniperpublishers.com/online-submission.php 